\title{
Amorphous to crystalline phase transition in carbon induced by intense femtosecond x-ray free-electron laser pulses
}

\author{
J. Gaudin,,${ }^{1, *}$ O. Peyrusse, ${ }^{2}$ J. Chalupský,,${ }^{3,4}$ M. Toufarová,,${ }^{3,4}$ L. Vyšín,,${ }^{3}$ V. Hájková, ${ }^{3}$ R. Sobierajski, ${ }^{5,6}$ T. Burian, ${ }^{3}$ \\ Sh. Dastjani-Farahani, ${ }^{1}$ A. Graf, ${ }^{7}$ M. Amati, ${ }^{8}$ L. Gregoratti, ${ }^{8}$ S. P. Hau-Riege, ${ }^{7}$ G. Hoffmann, ${ }^{9}$ L. Juha, ${ }^{3}$ J. Krzywinski, ${ }^{10}$ \\ R. A. London, ${ }^{7}$ S. Moeller, ${ }^{10}$ H. Sinn, ${ }^{1}$ S. Schorb, ${ }^{10}$ M. Störmer, ${ }^{11}$ Th. Tschentscher, ${ }^{1}$ V. Vorlíček, ${ }^{3}$ \\ H. Vu, ${ }^{9}$ J. Bozek, ${ }^{10}$ and C. Bostedt ${ }^{10}$ \\ ${ }^{1}$ European XFEL GmbH, Albert-Einstein-Ring 19, D-22761 Hamburg, Germany \\ ${ }^{2}$ Université de Bordeaux-CNRS-CEA, Centre Lasers Intenses et Applications, F-33405 Talence, France \\ ${ }^{3}$ Institute of Physics, Academy of Sciences of the Czech Republic, Na Slovance 2, 18221 Prague 8, Czech Republic \\ ${ }^{4}$ Faculty of Nuclear Sciences and Physical Engineering, Czech Technical University in Prague, Břehová 7, 115 19 Prague 1, Czech Republic \\ ${ }^{5}$ Institute of Physics PAS, Al. Lotników 32/46, PL-02-668 Warsaw, Poland \\ ${ }^{6}$ Dutch Institute for Fundamental Energy Research, PO Box 1207, 3430 BE Nieuwegein, The Netherlands \\ ${ }^{7}$ Lawrence Livermore National Laboratory, Livermore, California 94550, USA \\ ${ }^{8}$ Sincrotrone Trieste SCpA, SS14-Jm Area Science Park, 34149 Trieste, Italy \\ ${ }^{9}$ Institute of Applied Physics, University of Hamburg, D-20355 Hamburg, Germany \\ ${ }^{10}$ SLAC National Accelerator Laboratory, 2575 Sand Hill Road, Menlo Park, California 94025, USA \\ ${ }^{11}$ Helmholtz Zentrum Geesthacht, Institute of Materials Research, Max-Planck-Strasse 1, D-21502 Geesthacht, Germany
}

(Received 7 May 2012; published 11 July 2012)

\begin{abstract}
We present the results of an experiment where amorphous carbon undergoes a phase transition induced by femtosecond $830 \mathrm{eV}$ x-ray free-electron laser pulses. The phase transition threshold fluence is found to be $282 \pm 11 \mathrm{~mJ} / \mathrm{cm}^{2}$. Atomic force microscopy, photoelectron microscopy, and micro-Raman spectroscopy give experimental evidence for the phase transition in terms of a volume expansion, graphitization, and change of local order of the irradiated sample area. The interaction is modeled by an accurate time-dependent treatment of the ionization dynamics coupled to a two-temperature model. At the phase transition fluence threshold the free-electron density $N_{e}$ is found to be at maximum $9 \times 10^{20} \mathrm{~cm}^{-3}$ while the ion (atom) temperature is found to be $1050 \mathrm{~K}$, e.g., above the crystallization activation temperature reported in the literature. This low ionization rate and high atom temperature suggest a thermally activated phase transition.
\end{abstract}

DOI: 10.1103/PhysRevB.86.024103

PACS number(s): 64.60.Cn, 64.70.dg, 61.80.Cb, 61.43.-j

\section{INTRODUCTION}

The advent of x-ray free-electron lasers (XFELs), also known as fourth-generation X-ray light sources, enables the study of interaction of matter with ultrashort, femtosecond, intense x-ray pulses. Despite the very recent commissioning of these facilities, prominent results have already been obtained in a wide range of scientific fields from atomic ${ }^{1}$ and molecular ${ }^{2}$ to plasma physics. ${ }^{3}$ Most of these studies have focused on the interaction of matter with ultraintense pulses. In the field of solid-state physics, the interaction regime below the threshold of plasma formation, which can be considered as the moderate-fluence regime, is also interesting as nondestructive solid to solid phase transitions can be triggered. A particularly interesting case is the amorphous to crystalline transition. From a fundamental point of view, these types of transitions allow studying the relation between local structures and physical properties. ${ }^{4}$ Furthermore they have also important practical implications, for example in data storage technology. ${ }^{5}$ Amorphous to crystalline transitions can be triggered by ultrashort optical laser pulses. ${ }^{6}$ In this latter case, the excitation of a dense electron-hole plasma leads to an ultrafast nonthermal phase transition occurring on a time scale faster than a thermally activated phase transition. In the x-ray domain the interaction of radiation with solids is in principle different compared to the optical domain as several additional processes are involved such as core level photoionization and Auger electron decay. As a result, various excitation and relaxation channels open up. It is then questionable whether amorphous to crystalline phase transitions can be induced by femtosecond X-ray pulses, and whether the origin of the transition is thermal or nonthermal. A first step to explore this new physics is to expose materials and characterize ex situ the effect of the irradiation. We report on such an experiment using a well-characterized material-amorphous carbon-as a test material.

Amorphous carbon (a-C) is a carbon synthetic allotrope, made of a mixture of tetrahedral $s p^{3}$ (diamond-like) and trigonal $s p^{2}$ (graphite-like) carbon in various ratios. For high $s p^{3}$ content, this material is called diamond-like carbon. Its physical properties (such as mechanical and radiation hardness, biocompatibility, and chemical inertness) make it of high relevance in numerous domains ranging from $\mathrm{X}$-ray optics ${ }^{7}$ to microelectronics (see Ref. 8 for a comprehensive review). Phase transitions in a-C have previously been investigated using different types of triggering processes: annealing ${ }^{9-11}$ and irradiation by ions, ${ }^{12}$ electrons,${ }^{13}$ and optical laser pulses. ${ }^{14}$ All studies demonstrated that the a-C structure tends to be modified to a more ordered graphitic phase, e.g., by undergoing an amorphous $\rightarrow$ crystalline transition.

We present the results of an experiment conducted at the Linac Coherent Light Source (LCLS), ${ }^{15}$ where a-C was irradiated by a $830 \mathrm{eV}$ x-ray FEL beam. In the first part of this article, we describe the experiment and the procedure used to determine the phase transition energy threshold by 

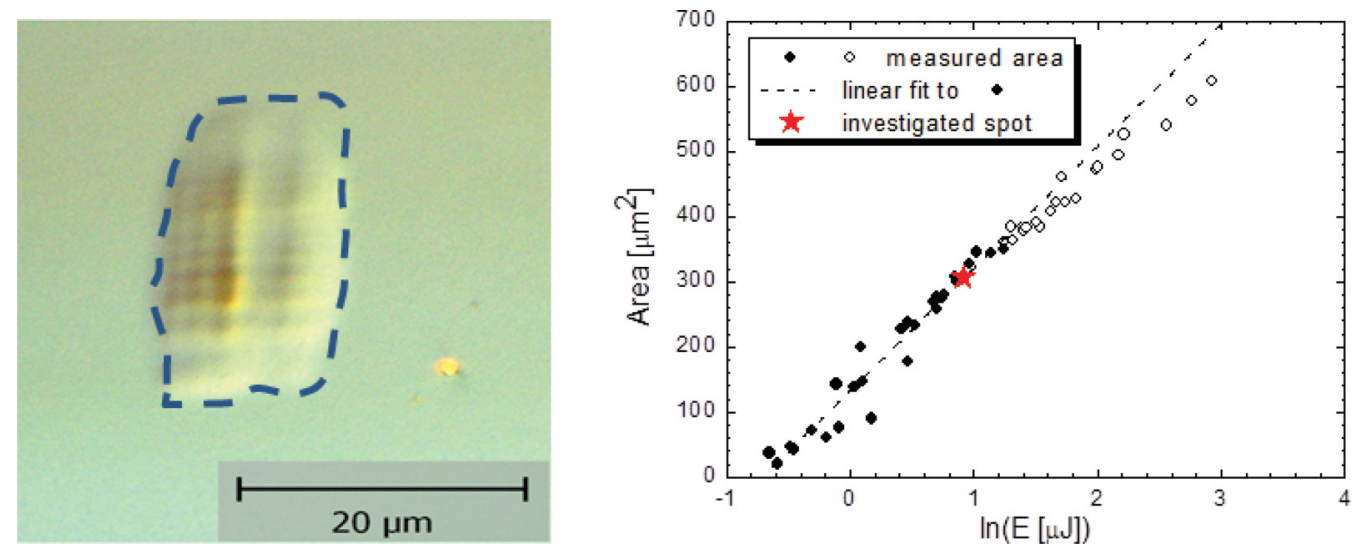

FIG. 1. (Color online) Left: Typical DIC image. The contour corresponds to the area measured. Right: Surface area of the irradiated materials as measured with DIC microscope as a function of impinging energy. The red star corresponds to the spot shown in the left part of this figure.

using ex situ optical microscopy. The second part focuses on scanning photoelectron microscopy (SPEM) and microRaman spectroscopy analysis. These two analysis tools are complementary as SPEM provides information on the chemical bonding while Raman spectroscopy unravels short-range atomic order. The third part describes the model developed to simulate the interaction of a femtosecond $\mathrm{x}$-ray pulse with a-C. The results give an estimation of the main parameters of the interaction: free-electron density and temperature as well as ion temperature. Finally, by synthesizing experimental and theoretical results, the question of whether material modification is of thermal or nonthermal nature is discussed.

\section{EXPERIMENT AND DETERMINATION OF PHASE TRANSITION ENERGY THRESHOLD}

The experiment was performed at the atomic, molecular, and optical science instrument at the LCLS facility. The $830 \mathrm{eV}$ photon beam was focused with a Kirkpatrick-Baez mirrors system onto the sample at normal incidence angle. The irradiation was performed under vacuum. After each laser pulse, the sample was moved to a fresh spot. The single-pulse energy on the sample ranged up to $30 \mu \mathrm{J}$ was measured with a gas monitor detector based on the calibrated measurement of the x-ray-induced fluorescence in $\mathrm{N}_{2}$. The energy per pulse was varied using a gas attenuator (all details on the beamline are given in Ref. 16). The pulse duration was approximately 60-100 fs. The sample consisted of a $1.4 \mu \mathrm{m}$ thick a-C coating on a Si substrate. The a-C was deposited by magnetron sputtering and $\mathrm{x}$-ray reflectivity measurements indicated a density of $2.2 \mathrm{~g} / \mathrm{cm}^{3},{ }^{17}$ corresponding to a ratio of $s p^{3} / s p^{2}$ of $0.2{ }^{8}$

In order to determine the phase transition threshold, we used optical microscopy with differential interference contrast (DIC), which is sensitive to variation of optical refractive index, hence to any phase change. For each irradiated sample region, the area (as shown in Fig. 1) on the microscope image was measured. Figure 1 shows the dependence of the area versus incident energy near the transition threshold. The x-ray-induced pattern directly images the contour of the focused laser beam, provided that the local energy is higher than the energy threshold for the phase transition. The area of the focus is a constant parameter; as the energy pulse increases, the enclosed area above the phase transition increases, as seen in Fig. 1.

The energy threshold is found to be $0.49 \pm 0.2 \mu \mathrm{J}$ by fitting the experimental points with a logarithmic function as described in Ref. 18. Due to the non-Gaussian shape of the beam, only the very first points (the filled-black ones in Fig. 1) are used for the fitting procedure. In order to determine the corresponding fluence threshold the beam area is needed. Since the spatial profile of the beam is non-Gaussian, a specific method described in detail in Ref. 19 has been applied to retrieve the effective area, $A_{\text {eff }}$. This surface is equivalent to the area at the 1/e level of a Gaussian beam. The effective area is then found to be $174 \mu \mathrm{m}^{2}$, leading to a fluence threshold of $282 \pm 11 \mathrm{~mJ} / \mathrm{cm}^{2}$. Another useful quantity is the averaged surface dose absorbed per atom. Taking into account the photoabsorption cross section of $3706 \mathrm{~cm}^{2} / \mathrm{g},{ }^{20}$ the surface dose is found to be $131 \pm 5 \mathrm{meV} /$ atom. In comparison, this threshold is lower than for bulk $\mathrm{B}_{4} \mathrm{C}$ where the threshold was found to be $684 \mathrm{meV}$ /atom for similar irradiation conditions. ${ }^{21}$

Further insight is gained with atomic force microscopy (AFM) measurements. Figure 2(a) shows an example for an irradiation with incident energy of $2.5 \mu \mathrm{J}$ (red star in Fig. 1). It appears that the irradiated material undergoes a volume expansion and no ablation. The AFM measurements also provide an effective way to estimate the local fluence value, considering that each height is related to a fluence value. This method was utilized in Ref. 3 and is described in detail in the Appendix. The resulting fluence map is shown in Fig. 2(b).

\section{CHEMICAL AND STRUCTURAL ANALYSIS}

The irradiation by an XFEL pulse at moderate fluence triggers a solid to solid phase transition. In order to gain more insight into the nature of the modification, two types of analysis were performed ex situ: SPEM and micro-Raman analysis. SPEM provides information on the chemical bonding while Raman spectroscopy unravels short-range atomic order. 

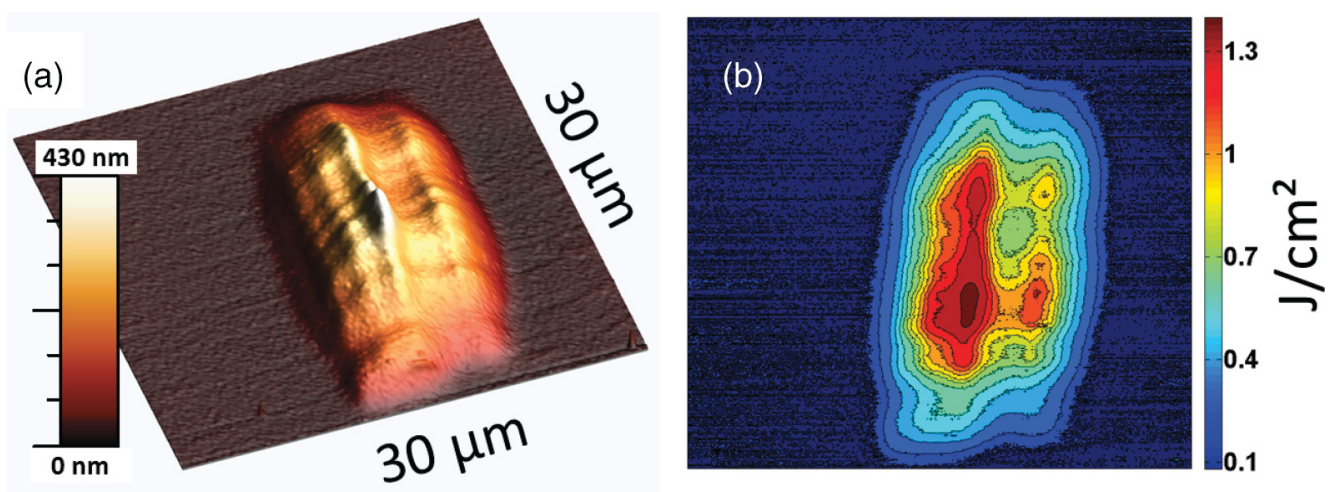

FIG. 2. (Color online) (a) AFM topography of an irradiated area with a $2.5 \mu \mathrm{J}$ FEL pulse. (b) Fluence map deduced from the AFM measurement. The color scale is in $\mathrm{J} / \mathrm{cm}^{2}$.

\section{A. Scanning photoelectron microscopy}

Photoemission spectroscopy (PES) is a well-established technique to characterize a-C $\mathrm{C}^{22,23}$ and its $s p^{3}$ and $s p^{2}$ content. SPEM as an imaging technique allows the same type of analysis in a space-resolved way. The experiment was performed at the ESCA microscopy beamline at the Elettra synchrotron facility. The complete description of the beamline is given in Ref. 24. Briefly, a $649 \mathrm{eV}$ x-ray beam is monochromatized and then focused on the sample with a zone plate optic, producing a circular spot with a diameter of about $150 \mathrm{~nm}$. The sample is then raster scanned with respect to the focused beam. The photoemitted electrons are then collected by a hemispherical sector spectrometer and measured by a multichannel detector. For each pixel, the acquisition is done in a spectroimaging mode, whereby the energy-dispersed electrons are measured by a 48-channel detector. As a result, the photoemission spectrum over a limited energy range is also measured for each point of the image. Figure 3 shows the $\mathrm{C} 1 \mathrm{~s}$ core level photoemission spectrum resulting from an average over $10 \times 10$ pixels for two different regions corresponding to



FIG. 3. Photoemission spectra from SPEM measurements obtained from averaging over two $10 \times 10$ pixel areas. Black curve: Nonirradiated sample. Gray curve: Center of the irradiated sample corresponding to $1.4 \mathrm{~J} / \mathrm{cm}^{2}$. a nonirradiated area and the area shown in Fig. 2, which is irradiated at a fluence of $1.4 \mathrm{~J} / \mathrm{cm}^{2}$. The content of $s p^{2}$ and $s p^{3}$ bonds can be deduced by fitting the peak with two components: one corresponding to $s p^{2}$ bonds around $284 \mathrm{eV}$, and a second one corresponding to $s p^{3}$ bonds at $0.9 \mathrm{eV}$ higher binding energy. ${ }^{22,23}$ The irradiation induces a shift of the spectrum towards lower binding energy and a slight amplitude decrease of the high binding energy part, similar to what has been observed from an annealed a-C sample. ${ }^{22}$

We define two energy ranges: the first one $(282.9 \mathrm{eV}$ to $284.7 \mathrm{eV}$ ) where the signal is mainly due to $s p^{2}$ bonds and the second one ( $284.9 \mathrm{eV}$ to $286.7 \mathrm{eV}$ ) corresponding to $s p^{3}$ bonds. The chemical information is extracted from the SPEM images in the following way: The images resulting from the integration of the signal over these particular ranges are divided by the image resulting from the sum of all the channels. The resulting images are shown in Fig. 4. The left figure corresponds to $s p^{2}$ and the right one to $s p^{3}$ content. In the irradiated area the $s p^{2}$ component is higher, whereas the $s p^{3}$ is reduced compared to the pristine material, indicating the conversion of the $s p^{3}$ to the $s p^{2}$ bonds, e.g., graphitization of the sample. Since the two components of the C $1 s$ peak overlap, the SPEM images give only a qualitative indication and not an absolute measurement of the hybrid contents.

\section{B. Micro-Raman analysis}

The micro-Raman analyses were performed prior to the SPEM measurements, in order to avoid any influence of the x-ray irradiation. The Raman spectra were measured in the usual backscattering geometry with a micro-Raman spectrometer (Renishaw Ramascope, model 1000) coupled to an optical microscope focusing the $\lambda=514.5 \mathrm{~nm}$ laser beam to a $2 \mu \mathrm{m}$ spot diameter. At this wavelength only the $s p^{2}$ bonds are probed, due to their 50 to 250 times higher scattering cross section as compared to $s p^{3} .^{25}$ Trial measurements were performed on test samples in order to determine the appropriate experimental conditions providing clean spectra without material modifications by laser annealing. Selected Raman spectra, corresponding to nonirradiated areas and areas irradiated at different fluences indicated in Fig. 2, are shown in Fig. 5. The spectra clearly show a broad asymmetrical feature typical of the a-C structure for the nonirradiated part. For the 

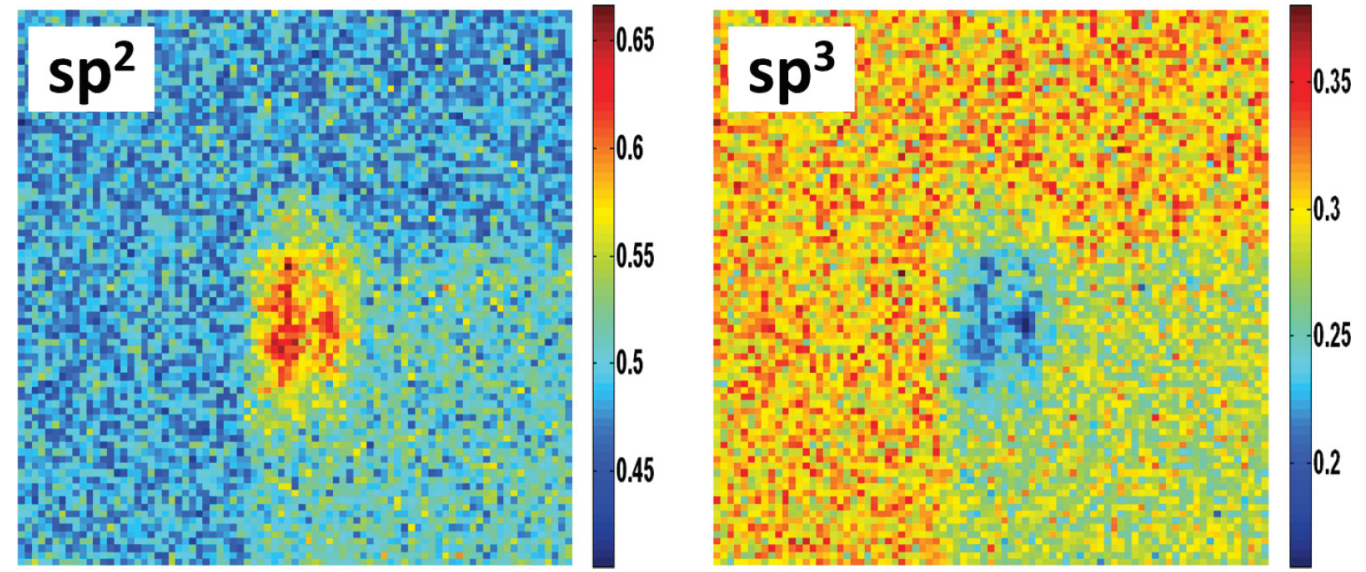

FIG. 4. (Color online) Left: SPEM measurement corresponding to the fraction of $s p^{2}$ bonds. Right: SPEM measurement corresponding to the fraction of $s p^{3}$ bonds. The square in the right bottom part of the pictures is the residual due to a previous scan measurement done to retrieve the exact location of the irradiated spot. The image dimensions are $89.6 \times 89.6 \mu \mathrm{m}$.

irradiated sample the two components of the initial feature clearly show up as the fluence increases. The first peak around $1350 \mathrm{~cm}^{-1}$ (D peak) is related to defect and breathing modes of $s p^{2}$ rings while the second peak around $1600 \mathrm{~cm}^{-1}$ (G peak) originates in the graphite related doubly degenerate phonon mode ( $E_{2 g}$ symmetry). The measured spectra are fitted using a Lorentzian line for the D peak and a Breit-Wigner-Fano (BWF) line for the G peak. ${ }^{26}$ The BWF line shape has the advantage of being equivalent to a Lorentzian shape when $1 / Q \rightarrow 0$, where $Q$ is the BWF coupling coefficient. The spectrum corresponding to initial and irradiated material can then be fitted with the same line shape as shown in Fig. 5.

Two quantities are extracted from the fitting curves, as shown in Fig. 5: the $\mathrm{G}$ peak position and the integrated peak intensity ratio $A(\mathrm{D}) / A(\mathrm{G})$. The right graph in Fig. 5 shows the displacement of the $G$ peak maximum from $1560 \mathrm{~cm}^{-1}$ for the nonirradiated material up to $1600 \mathrm{~cm}^{-1}$ at the maximum fluence, e.g., $1.4 \mathrm{~J} / \mathrm{cm}^{2}$. This can be explained by the decrease of the $s p^{2}$ bond length while the material turns to ordered graphite. This induces a bond strengthening and a phonon mode hardening reflected by a shift of the $\mathrm{G}$ peak. ${ }^{25}$ Graphite is made of stacked graphene layers. The in-plane size of these layers, denoted $L_{a}$, has been shown to be related to the $A(\mathrm{D}) / A(\mathrm{G})$ ratio. This ratio can be considered as a measurement of the degree of order in the a-C sample. Two relations are possible, depending on the structure of the material: ${ }^{26}$ For a-C (small ordered regions with $L_{a}<2 \mathrm{~nm}$ ) $A(\mathrm{D}) / A(\mathrm{G}) \sim L_{a}^{2}$, while for graphitic nano- and microcrystals $A(\mathrm{D}) / A(\mathrm{G}) \sim 1 / L_{a}$. The results shown in Fig. 5 can then be explained as follows: The $A(\mathrm{D}) / A(\mathrm{G})$ ratio increases as the beam fluence increases up to $0.6 \mathrm{~J} / \mathrm{cm}^{2}$ following the rule or trend for the a-C structure. For higher fluences, the value of $A(\mathrm{D}) / A(\mathrm{G})$ decreases indicating that the material is mainly composed of growing graphitic nanocrystals. Considering the relation given in Ref. 10 , for $1.4 \mathrm{~J} / \mathrm{cm}^{2}, L_{a}$ is approximately $15 \mathrm{~nm}$. This shows that the size of the graphite nanocrystals grows as the fluence increases.
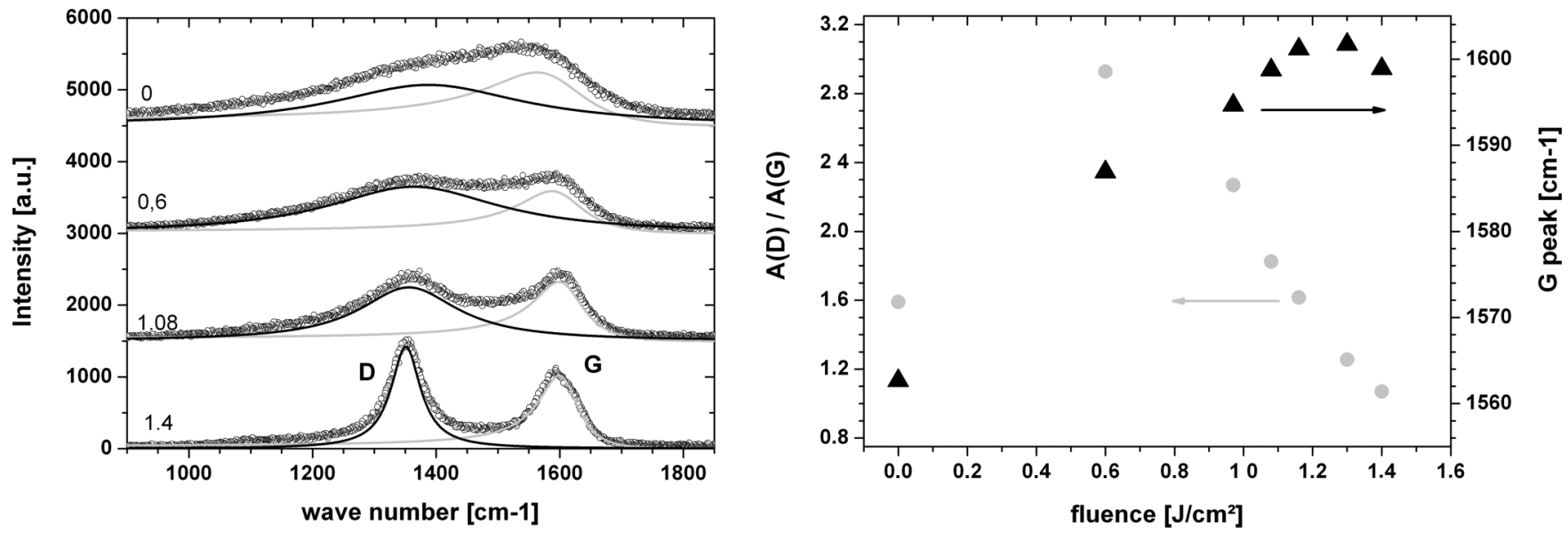

FIG. 5. Left: Selected Raman spectrum for different fluence (from top to bottom: 0, 0.6, 1.08, and $1.4 \mathrm{~J} / \mathrm{cm}^{2}$ ). The G (gray curve) and D (black curve) peaks used for the fitting are also shown. Right: Gray point: Area ratio of G and D peak resulting from the fit. Black triangles: Position of the $\mathrm{G}$ peak. 


\section{TWO-TEMPERATURE MODEL OF THE INTERACTION}

We describe here our model developed to simulate the $\mathrm{x}$-ray/matter interaction. Unlike molecular dynamics approaches, this model belongs to the class of continuum models. ${ }^{27}$ It is a one-fluid, two-temperature $\left(T_{e}, T_{i}\right)$ model. Ionization dynamics is described with a large set of timedependent rate equations, each of them describing a relevant bound electron configuration in the atomic and ionic species. In the $\mathrm{x}$-ray regime around $1 \mathrm{keV}$, and for light elements like carbon, the primary photon-matter interaction is photoionization
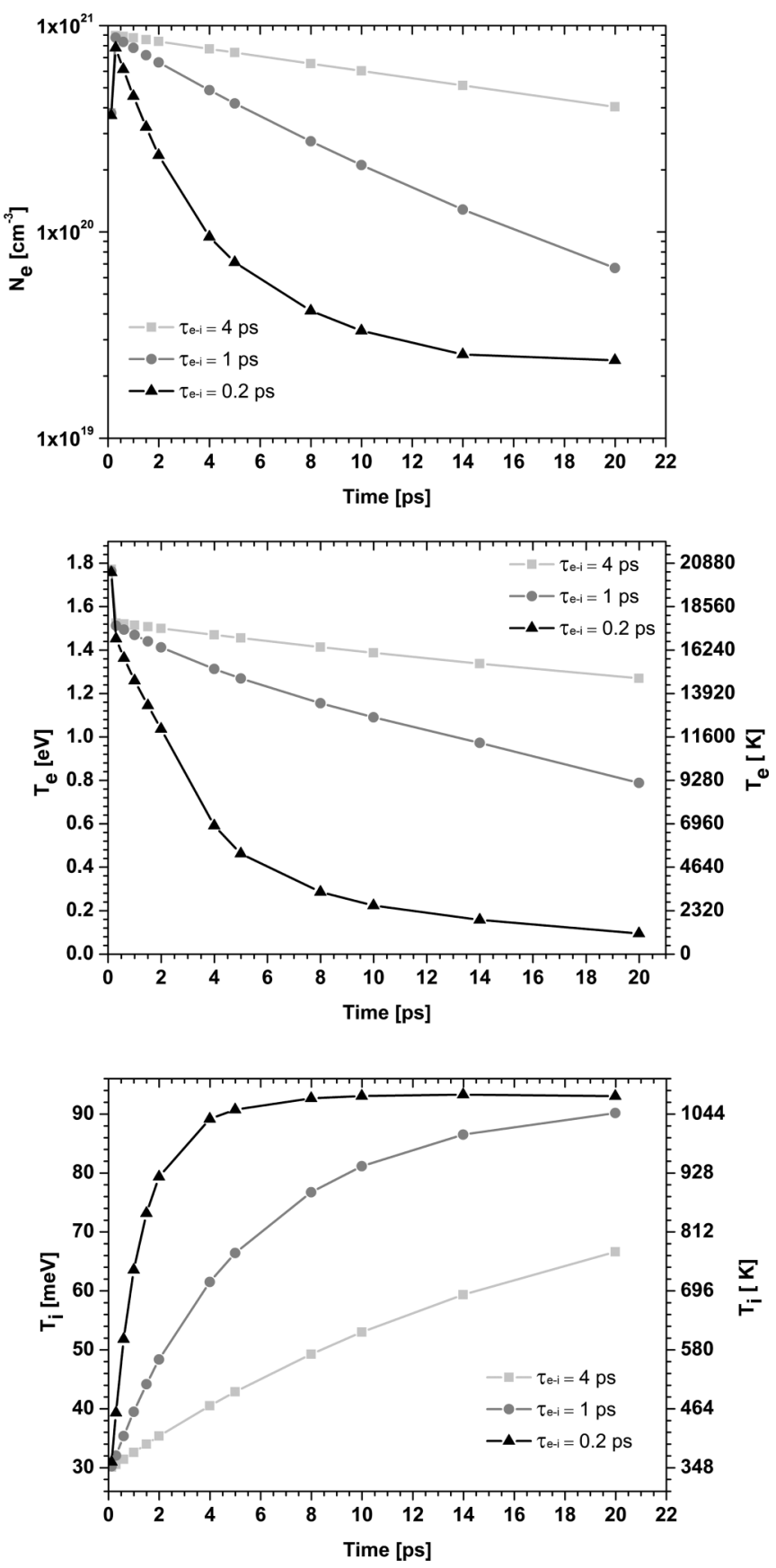

FIG. 6. Temporal evolution of $N_{e}$ (top), $T_{e}$ (middle), and $T_{i}$ (bottom). of the inner $K$-shell electrons. Then, the excited electrons relax through Auger decays giving secondary electrons. All of the excited free electrons thermalize quickly with each other and, in our model, thermalization is supposed to be instantaneous so that a temperature $\left(T_{e}\right)$ may be ascribed to the free electrons. Therefore, some of these electrons may contribute to further ionization through collisional processes. Processes included in the rate equation system mentioned above are photoionization, Auger decay and resonant capture, collisional ionization and recombination, collisional excitation and deexcitation, and radiation decay. Rates or cross-sections characterizing these couplings between the configurations are calculated properly using standard quantum mechanical methods, ${ }^{28}$ prior to performing the simulations. In our model, ion and electron temperatures are coupled through an equilibration time $\left(\tau_{e-i}\right)$ which has been set to a fixed value. To the best of our knowledge no value of $\tau_{e-i}$ in a-C has been measured or calculated. On the contrary, a large number of works have been devoted to graphite, which is very similar to the $s p^{2}$-rich a-C sample used in this experiment. An examination of the literature led us to consider a value of $\tau_{e-i}$ as between $200 \mathrm{fs}$ and 4 ps. $^{29-31}$

The experiment was modeled by considering a $1.4 \mu \mathrm{m}$ thick layer of carbon of $2.2 \mathrm{~g} / \mathrm{cm}^{-3}$ density. The pulse duration was fixed to FWHM $=100 \mathrm{fs}$. The simulation were performed for 3 values of $\tau_{e-i}=0.2,1$, and 4 ps. Temporal evolutions of $N_{e}$, $T_{e}$, and $T_{i}$ at the sample's surface in the case of a fluence equal to $300 \mathrm{~mJ} / \mathrm{cm}^{2}$, corresponding to the measured fluence phase transition threshold, are shown in Fig. 6.

The results in Fig. 6 show two remarkable features. The first one is the very steep coupled evolution of $N_{e}$ and $T_{e}$ after the laser pulse during the first $300 \mathrm{fs}$. As can be seen $N_{e}$ drastically increases while $T_{e}$ decreases. This can be understood as the effect of the excitation of high-energy photoelectrons and Auger process leading to the excitation of secondary electrons, hence the increase of $N_{e}$, and a subsequent decrease of $T_{e}$ as the total energy has to be conserved. The second feature concerns the ion temperature $T_{i}$. For $\tau_{e-i}=0.2 \mathrm{ps}$ and $1 \mathrm{ps}$, this temperature saturates to the same final value $T_{i}^{f} \approx 1050 \mathrm{~K}$, independently of the value of $\tau_{e-i}$. The model shows that the

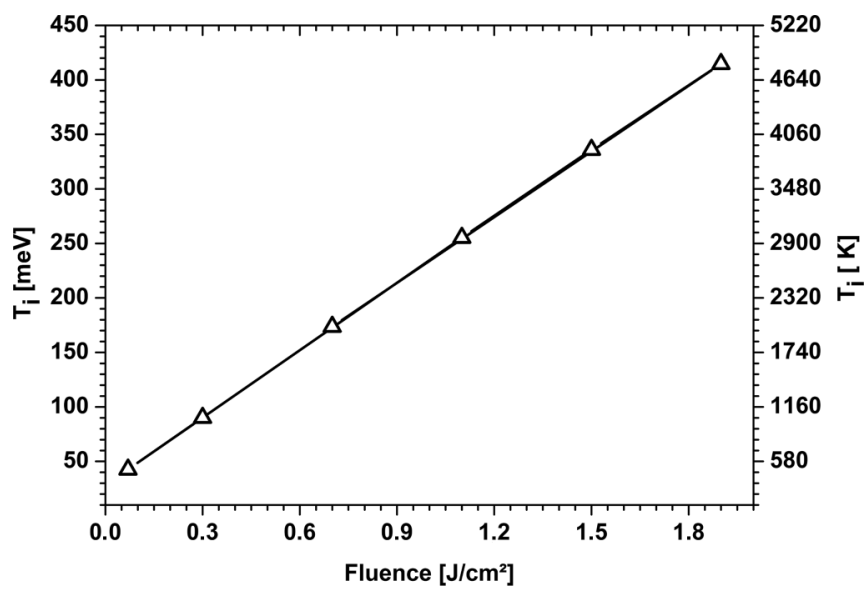

FIG. 7. Triangles: Values of $T_{i}$ at $20 \mathrm{ps}$ obtained for $\tau_{e-i}=1 \mathrm{ps}$. The line is a linear fit of the data. 
main part of the energy absorbed by the electronic system is then passed on to atoms, being converted in heat. There is negligible loss of energy due to radiative processes. We infer that for $\tau_{e-i}=4 \mathrm{ps}$, the value of $T_{i}^{f}$ obtained also reaches the same value $T_{i}^{f} \approx 1050 \mathrm{~K}$, but on a time scale somewhat longer than our simulations. Several simulations were performed to investigate the evolution of $T_{i}^{f}$ as a function of fluence, only for the case of $\tau_{e-i}=1 \mathrm{ps}$. The values of $T_{i}^{f}$ at $20 \mathrm{ps}$ are plotted in Fig. 7, showing a linear behavior.

\section{DISCUSSION}

We shall now relate the experiment and simulation results to gain deeper insight into the origin of the phase transition. Covalent materials, like carbon, have been shown to undergo ultrafast nonthermal phase transition while irradiated by fs optical pulses. The basic picture is that the high-density electron-hole plasma excited by the laser pulse modifies the potential energy surface. In the extreme case the energy barrier between the two phases involved is canceled, which triggers a pure nonthermal phase transition on the sub-ps time scale. Theory ${ }^{32}$ and experiments ${ }^{33}$ have shown that the driving parameter in this type of transition is the free-electron density. A minimum value of around $10 \%$ of the valence band electrons is required for a nonthermal process to occur. In the case of a-C, the minimum free-electron density is then $N_{e}^{\text {min }} \cong 4 \times 10^{22} \mathrm{~cm}^{-3}$. The highest value of $N_{e}$ provided by the simulations is $N_{e}=9 \times 10^{20} \mathrm{~cm}^{-3} \ll N_{e}^{\min }$, which rules out a purely nonthermal phase transition.

For lower excitation density the energy barrier is only reduced. In this case, the phase transition needs to be thermally activated, but the temperature activation is lowered compare to a purely thermally induced phase transition. A number of annealing experiments have been performed which can be compared to our results to evaluate the photon-induced energy barrier reduction consequence. Two threshold temperatures can be distinguished: The first one corresponds to the onset of the phase transition, i.e., amorphous structure ordering in graphite nanocrystals. The second one corresponds to the conversion of $s p^{3}$ bonding to $s p^{2}$. This second threshold in our experiments is estimated to be $420 \pm 40 \mathrm{~mJ} / \mathrm{cm}^{2}$, by comparing the fluence map of Fig. 2 and the SPEM image in Fig. 4. The corresponding temperatures are obtained via the simulations, namely the line shown in Fig. 7. Experimental results taken from Refs. 9 and 11, which used various diagnostic techniques, are presented in Table I. Both temperature thresholds are similar for the two references, indicating that these temperatures are only slightly dependent on the $s p^{3} / s p^{2}$ and deposition techniques (also indicated in Table I), and can be used for our specific a-C sample. The values in Table I show that both threshold temperatures found for our experiment correspond to temperatures obtained by annealing of a-C. This indicates the negligible influence of the nonthermal effect, and the pure thermal activation of the phase transition.

\section{CONCLUSION}

We have measured the fluence threshold for phase transition in a-C induced by femtosecond XFEL pulses to be $282 \pm$
TABLE I. Comparison of annealing experiments for different types of a-C (PLD: pulsed laser deposition, FCVA: filtered cathodic vacuum arc). The experimental techniques used to determine the temperature are given in parentheses.

\begin{tabular}{lccc}
\hline \hline Ref. & $\begin{array}{c}\text { Initial } \\
s p^{3} / s p^{2}\end{array}$ & $\begin{array}{c}\text { Threshold } T \text { of } \\
\text { phase transition }\end{array}$ & $\begin{array}{c}\text { Threshold } T \\
\text { for } s p^{3} \rightarrow s p^{2}\end{array}$ \\
\hline 12 & 0.1 & $900 \mathrm{~K}$ & $1600 \mathrm{~K}$ \\
10 & (PLD) & (x-ray diffraction) & (electron energy loss) \\
& 0.8 & $1000 \mathrm{~K}$ & $1300 \mathrm{~K}$ \\
This work & 0.2 & $1050 \mathrm{~K}$ & (UV Raman) \\
\hline \hline
\end{tabular}

$11 \mathrm{~mJ} / \mathrm{cm}^{2}$. At low fluence the initial $s p^{2}$ bonded atoms start to form graphite nanocrystals as evidenced by the micro-Raman analysis. The analysis also shows that as the incident fluence is increased, the in-plane nanocrystal size becomes larger. The SPEM analyses demonstrate the conversion of the initial $s p^{3}$ bonds to $s p^{2}$ bonds. The model developed to simulate the interaction of femtosecond $\mathrm{x}$-ray pulses with carbon shows on the one hand that the excitation density is not high enough to trigger a nonthermal transition. On the other hand, comparison of the ion temperatures provided by the simulation with previous annealing experiment indicates a negligible influence of the photon-induced effects. Two reasons can be brought up: The density of excitation is either too low or too short-lived to influence the phase transition activation. Finally, combining the theoretical and experimental results provide compelling evidence of the pure thermal activation of the phase transition. Our experiment is a first step towards the understanding of interaction of intense $\mathrm{x}$-ray pulse with solids in the moderate-intensity level. This experiment also shows that the solid to solid transition can be triggered by intense $\mathrm{x}$-ray pulses paving the way to time-resolved experiments enabling the investigation of crystallization process on the fs to ps time scale.

\section{ACKNOWLEDGMENTS}

Portions of this research were carried out at the Linac Coherent Light Source (LCLS) at the SLAC National Accelerator Laboratory. LCLS is an Office of Science User Facility operated for the US Department of Energy Office of Science by Stanford University.

\section{APPENDIX}

The full mathematical development of the method used to calculate the fluence map is given in Ref. 19. The qualitative description given in this paragraph should enable the reader to understand the underlying idea of this method. The fluence map in Fig. 2 relies primarily on the assumption that there is no transverse energy transfer. As results we can assign the isoheight contours and their respective areas a fluence value, knowing the total pulse energy and the so-called fluence scan (f-scan) of the beam profile. ${ }^{19}$ The f-scan function $f(S)$ puts into relation the local fluence $F$ and the corresponding beam 


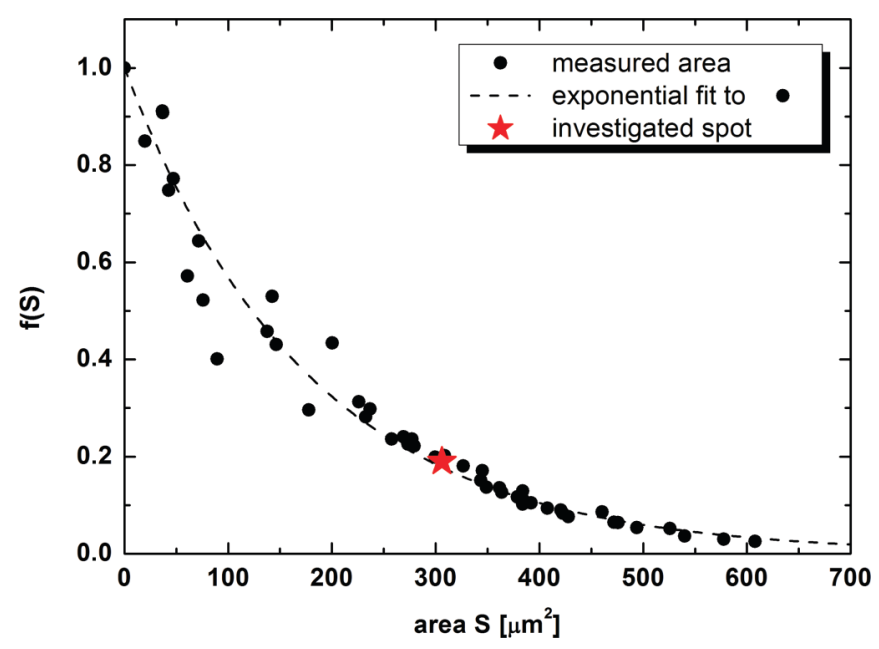

FIG. 8. (Color online) Fluence scan of the LCLS beam profile used for sample irradiation. The data (closed black circles) are fitted by an exponential function (black dashed line). The investigated spot is indicated by a red asterisk.

cross-section (contour) area $S$ via the expression $F(S)=F_{0}$ $\times f(S)$. Here, $F_{0}=E / A_{\text {eff }}$ is the real peak fluence calculated as a ratio between the actual pulse energy $E$ and the effective beam area $A_{\text {eff }}$. According to Ref. 19, the $f(S)$ function can be evaluated from the data depicted in Fig. 1 and is plotted in Fig. 8.

Furthermore, the fluence scan was fitted by an exponential function which in turn gives an approximate relation between

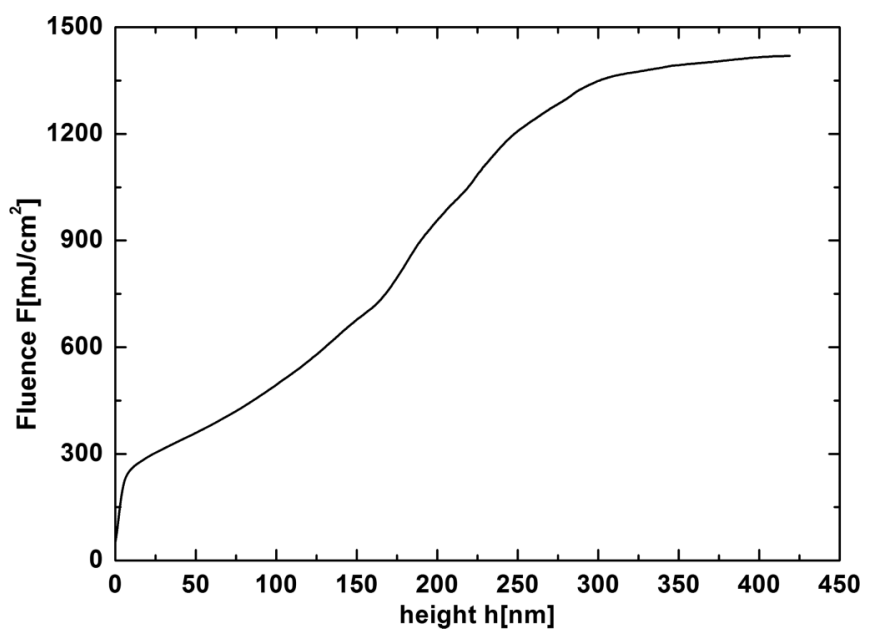

FIG. 9. Calibration curve relating local fluence and height.

the local fluence and the corresponding contour area: $F(S)=$ $F_{0} \exp \left(-S / A_{\text {eff }}\right)$. From the AFM morphology of the expanded structure in Fig. 2(a) the isoheight contours can be easily retrieved and, therefore, the relation between heights $h$ and the corresponding contour areas $S$ can be determined. Hence it follows that one height value corresponds to one local fluence value being linked via the contour area. Consequently, we obtain a calibration curve (see Fig. 9) putting into relation the local height and fluence within a confidence interval of $20 \%$.
*Corresponding author: jerome.gaudin@xfel.eu

${ }^{1}$ L. Young et al., Nature (London) 466, 56 (2010).

${ }^{2}$ M. Hoener et al., Phys. Rev. Lett. 104, 253002 (2010).

${ }^{3}$ S. M. Vinko et al., Nature (London) 482, 59 (2012).

${ }^{4}$ W. Welnic et al., Nat. Mater. 5, 56 (2006).

${ }^{5}$ S. Raoux, W. Welnic, and D. Lelmini, Chem. Rev. 110, 240 (2010).

${ }^{6}$ K. Sokolowski-Tinten et al., Phys. Rev. Lett. 81, 3679 (1998).

${ }^{7}$ J. Chalupský et al., Appl. Phys. Lett. 95, 031111 (2009).

${ }^{8}$ J. Robertson, Mater. Sci. Eng. R 37, 129 (2002).

${ }^{9}$ A. C. Ferrari et al., J. Appl. Phys. 85, 7191 (1999).

${ }^{10}$ L. G. Cançado et al., Appl. Phys. Lett. 88, 163106 (2006).

${ }^{11}$ T. Takai et al., Phys. Rev. B 67, 214202 (2003).

${ }^{12}$ D. C. McCulloch et al., Phys. Rev. B 52, 850 (1995).

${ }^{13}$ Y. Linang, Y. Mera, and K. Maeda, Diamond Relat. Mater. 17, 137 (2008).

${ }^{14}$ T. V. Kononenko et al., Appl. Phys. A 79, 543 (2004).

${ }^{15}$ P. Emma et al., Nature Photon. 4, 641 (2010).

${ }^{16} \mathrm{~S}$. Moeller et al., Nucl. Instrum. Methods A 635, S6 (2011).

${ }^{17}$ M. Störmer, F. Siewert, and J. Gaudin, Proc. SPIE 8078, 80780G (2011).
${ }^{18}$ J. M. Liu, Opt. Lett. 7, 196 (1982).

${ }^{19}$ J. Chalupský et al., Opt. Express 18, 27836 (2011).

${ }^{20}$ Center for X-Ray Optics, http://henke.lbl.gov/optical_constants/.

${ }^{21}$ S. P. Hau-Riege et al., Opt. Express 18, 23933 (2010).

${ }^{22}$ J. Díaz et al., Phys. Rev. B 54, 8064 (1996).

${ }^{23}$ R. Haerle et al., Phys. Rev. B 65, 045101 (2001).

${ }^{24}$ M. Kazemian Abyaneh et al., e-J. Surf. Sci. Nanotech. 9, 158 (2011).

${ }^{25}$ A. C. Ferrari and J. Robertson, Phil. Trans. R. Soc. London A 362, 2477 (2004).

${ }^{26}$ A. C. Ferrari and J. Robertson, Phys. Rev. B 61, 14095 (2000).

${ }^{27}$ S. P. Hau-Riege, AIP Conf. Proc 1161, 100 (2009).

${ }^{28}$ O. Peyrusse, J. Phys. B 32, 683 (1999).

${ }^{29}$ M. Breusing, C. Ropers, and T. Elsaesser, Phys. Rev. Lett. 102, 086809 (2009).

${ }^{30}$ M. E. Garcia and H. O. Jeschke, Appl. Surf. Sci. 208, 61 (2003).

${ }^{31}$ P. L. Silvestrelli and M. Parrinello, J. Appl. Phys. 83, 2478 (1998).

${ }^{32}$ K. H. Bennemann, Ann. Phys. 18, 480 (2009).

${ }^{33}$ S. K. Sundaram and E. Mazur, Nat. Mater. 1, 219 (2002). 